\title{
L'instrumentation automatique en biologie moléculaire
}

\author{
Frédéric Ginot
}

\section{L'instrumentation en biologie} moléculaire change rapidement, le moteur principal de cette évolution étant la nécessité de produire plus. Cela est vrai pour les laboratoires académiques comme pour les grands Genome Centers ou les laboratoires privés. Le gain de productivité procède

essentiellement de deux éléments : l'augmentation de la cadence d'opérations répétitives et la diminution du temps passé en préparations et mises au point de techniques connues par ailleurs.

L'automatisation des opérations de routine peut, soit conserver les étapes des procédés manuels, soit modifier ces techniques pour pouvoir les automatiser. Les "stations de travail", appareils automatisant les fonctions élémentaires que sont le pipettage et les incubations, en sont encore à leur début, réservées aux laboratoires dont l'activité est focalisée sur un très petit nombre de techniques. Les laboratoires conventionnels seront intéressés dès que leur programmation sera simplifiée : les possibilités nouvelles apportées par l'instrumentation et le traitement informatisé des données modifient déjà considérablement le visage de la recherche.
Des instruments dans les laboratoires La biologie moléculaire, comme toute discipline scientifique nouvelle, a démarré avec des moyens expérimentaux très modestes : du papier buvard, de l'agarose, une alimentation stabilisée, et quelques centrifugeuses. Les bancs d'expérience reposaient essentiellement sur l'astuce et l'habileté manuelle des pionniers de cette science. Mais avec l'expansion qu'elle a connue durant les années 1980, de nombreux appareils sont apparus sur le marché, plus rapides et plus fiables que les bricolages de laboratoire. Même si la valeur de ces appareils est encore modeste en regard de celle des appareils présents dans les laboratoires d'autres disciplines scientifiques (chimie, physique nucléaire), elle représente désormais un poste de dépenses important. La valeur globale du parc des appareils dans les laboratoires de biologie moléculaire a donc augmenté au cours de ces dernières années [1].

Cette évolution n'est pas spécifique de la biologie, mais son caractère spectaculaire et la rapidité à laquelle se succèdent les techniques d'investigation fascinent les uns... et effraient les autres. Cet article se propose simplement de livrer quelques réflexions sur cette évolution, et d'avancer certaines hypothèses sur le futur proche de l'instrumentation automatique dans les laboratoires de biologie moléculaire.

Le moteur principal de cette évolution : produire plus

Il me semble pertinent d'appréhen- der la question de l'instrumentation en biologie à l'aide d'une clé de lecture simple, celle de la productivité. La notion de production en recherche reste controversée et difficile à définir [2], mais elle correspond néanmoins à une réalité. Il y a bel et bien production de connaissances, de savoir-faire... et de satisfactions, personnelles ou nationales !

De ce fait, les laboratoires de recherche sont évalués à leur "production ", même si nul ne sait la quantifier de façon satisfaisante. De là, et de la nature finie des crédits alloués à la recherche, découle une situation de compétition entre les laboratoires qui est particulièrement forte aujourd'hui dans les domaines «chauds " de la biologie moléculaire. Il n'est pas question dans cet article de se pencher sur les conséquences de cette situation, dont certaines sont bénéfiques, et d'autres perverses. Mais il importe d'être conscient de cette situation qui pousse les laboratoires à ce qui peut bien être appelé des gains de productivité. L'évolution de la consistance des articles scientifiques dans les grandes revues (Cell par exemple) est une illustration sans doute fidèle des gains de productivité qui ont été acquis au cours des dix dernières années. Le clonage d'un ADNc méritait à l'époque une publication entière [3, 4]. Aujourd'hui, quand il s'agit d'un facteur transcriptionnel, une publication qui se respecte ne contient pas seulement le clonage de l'ADNc. Il y est ajouté son séquençage, la comparaison de cette 
séquence à toutes celles contenues dans les bases de données, des caractérisations de la liaison du facteur, et des expériences fonctionnelles par transfection, quand ce n'est pas la production d'anticorps reconnaissant le facteur produit auparavant par génie génétique, et le profil tissulaire d'expression au niveau protéique. Des travaux récents comme ceux de Hake et Richter [5] ou de Schaffer et Lodish [6] montrent bien la diversité des techniques apparaissant désormais dans une seule publication.

Cet exemple sur le contenu des articles scientifiques indique clairement que l'ensemble des laboratoires de biologie moléculaire est concerné par cette quête de gains de productivité. Cela ne concerne pas seulement les gros centres de la génétique humaine du type Généthon ou Human Genome Center américain. Les laboratoires traditionnels y sont tout autant impliqués, même si l'évolution y est moins médiatisée.

Choisir comme clé de lecture la recherche de gains de productivité ne signifie pas que celle-ci permettra de tout dire sur la question de l'instrumentation en biologie. Bien d'autres aspects de cette question ne seront pas pris en compte dans cette approche, tant du côté du fabricant d'appareils de laboratoire que de celui de l'utilisateur. Du côté des fabricants par exemple, on peut citer la lourdeur des investissements nécessaires au développement d'un appareil automatique. Elle limite le nombre de développements qu'il est possible de mener en parallèle, et tend donc à freiner la progression de l'instrumentation. Cela est d'autant plus vrai que les techniques mises en jeu évoluent vite, et que les industriels craignent le développement d'appareils obsolètes dès leur lancement commercial. Du côté utilisateur, il existe des facteurs humains importants jouant un rôle dans la progression de l'instrumentation. On observe ainsi depuis des années dans les laboratoires de recherche une augmentation de la qualification du personnel. Le laborantin corvéable à merci est devenu rarissime ; on embauche plus volontiers des cher-

derniers sont de plus en plus qualifiés. Cette tendance s'observe autant aux États-Unis qu'en Europe, dans les laboratoires privés comme dans les laboratoires publics. La biologie étant avant tout une science expérimentale, les chercheurs travaillent donc beaucoup «à la paillasse ». Cela a des avantages indéniables quant à la conception et à l'interprétation des expériences, mais aussi un inconvénient majeur. Car les chercheurs, accaparés par des tâches matérielles parfois très répétitives, utilisent finalement peu leurs capacités d'analyse, de synthèse et d'imagination. Il existe donc un désir de libérer ces personnes des tâches "vulgaires" pour qu'elles puissent mieux se consacrer au travail pour lequel elles ont été formées et appelées. L'instrumentation permet de répondre en partie à ce besoin.

\section{Où gagner de la productivité ?}

Sur quelles opérations un laboratoire peut-il gagner de la productivité ? Une observation du travail de laboratoire permet de distinguer deux façons - non exclusives - d'atteindre cet objectif : l'augmentation de la cadence d'opérations répétitives, et la diminution du temps passé en préparations et mises au point de techniques connues par ailleurs.

1. Augmentation de la cadence d'opéra tions répétitives. La biologie moléculaire met en œuvre un grand nombre de techniques qui évoluent vite. Il existe cependant des opérations de base qui sont constamment utilisées. Celles-ci couvrent la préparation des échantillons (purification d'ADN génomique, plasmidique..., purification d'ARNm, amplification) et certaines analyses (toutes les techniques de buvardage (blotting), l'analyse d'image, le séquençage de l'ADN). On peut également noter que le cœur des expériences revient souvent à une série de pipettage et d'incubations, susceptible d'automatisation.

L'augmentation des cadences de ces opérations de base intéresse en premier lieu les gros projets, comme les projets génomes ou les recherches génétiques. En effet, elle rend possible certaines approches expérimen- tales, qui seraient autrement inaccessibles. L'archétype français de ce type d'approche est le Généthon ; sans une augmentation systématique des cadences de chaque étape d'un procédé de base, les projets de cartographie auraient pris quelques dizaines d'années au lieu d'être réalisés en quelques années [7, 8].

Mais cette augmentation des cadences intéresse aussi le laboratoire plus conventionnel: quand sa recherche nécessite le séquençage de quelques kilobases d'ADN, il est préférable que cela soit réalisé en une ou deux semaines plutôt qu'en six mois. De même, le clonage d'une entité rare (un ARNm peu abondant ou un mutant bien spécifique par exemple) n'a une bonne probabilité d'aboutir que si l'on dispose d'un moyen permettant le criblage d'un grand nombre de clones.

2. Diminution du temps passé en préparations et mises au point. Le déroulement d'un programme de recherche nécessite aujourd'hui d'utiliser des techniques très variées, certaines facilement accessibles, d'autres nécessitant un appareillage spécial (ex. : cytométrie de flux et trieur de cellules) ou une grande expérience (ex. : hybridation in situ). Ces techniques relèvent de domaines scientifiques parfois très différents, comme ceux de la biologie moléculaire, de la biologie cellulaire, de la microbiologie, de la biochimie, de l'enzymologie, etc. Un chercheur imaginatif cherchera donc à utiliser telle ou telle technique qui lui paraît adaptée à son besoin, mais ne peut plus être un spécialiste de toutes ces techniques. Cela est vrai, même pour les techniques courantes comme le séquençage de l'ADN: quand le besoin est de séquencer un ou deux fragments d'ADN tous les six mois, il n'est pas possible d'être un spécialiste des réactions et des gels de séquence.

Il y a donc une demande de solutions "clé en main" qui permettent une réduction significative des délais pour la réalisation d'expériences ponctuelles : il semble en effet absurde de passer des semaines à mettre au point dans son laboratoire des techniques utilisées quotidienne- 
ment dans d'autres laboratoires; quelques jours doivent désormais suffire.

Cette question du temps passé en mises au point expérimentales est cruciale pour tous les laboratoires. Mais elle l'est encore plus pour les laboratoires qui incluent le coût de la main-d'œuvre dans le prix de revient d'un résultat scientifique. Car le gain de productivité ne résulte pas alors seulement de l'accélération des expériences, mais aussi d'une baisse du coût total des expériences (qui inclut la main-d'œuvre et les réactifs). Et cela est d'autant plus vrai que la main-d'œuvre est plus qualifiée.

Comment gagner de la productivité ? L'augmentation de la cadence d'opérations répétitives et la diminution des temps de mises au point sont donc les deux cibles que l'on doit d'abord viser pour réaliser ce que nous avons appelé des gains de productivité. Mais comment atteindre ces cibles ? Deux moyens sont couramment utilisés aujourd'hui pour "produire plus»: l'utilisation de trousses manuelles, et l'instrumentation automatique. Ces deux moyens ont chacun leurs avantages et leurs inconvénients, mais ils tendent tous les deux vers le même but : rendre les expériences plus rapides, plus faciles et moins prenantes pour l'opérateur.

1. Les kits manuels. Depuis la fin des années 1980, des trousses (kits) sont apparues pour faciliter la vie de l'expérimentateur, en lui évitant les préparations fastidieuses de tampons. Ces trousses offrent tous les réactifs, prêts à l'emploi, nécessaires à la réalisation d'une opération de routine : marquage de l'ADN avec un isotope radioactif, réaction de séquences de l'ADN, transcription d'un ADN par une ARN-polymérase, purification d'un plasmide à partir d'une culture bactérienne, etc. De plus, ces trousses offrent en général un protocole expérimental éprouvé. L'utilisateur a donc tout sous la main pour son expérience, sans temps de préparation. Cette facilité de réalisation des opérations de base est extrêmement appréciée des expérimentateurs, et des trousses sont désormais utilisées dans tous les laboratoires.

Puisque les trousses manuelles offrent en général un protocole expérimental fiable et des réactifs prêts à l'eıploi, on comprend facilement qu'clles sont en général bien adaptées aux gains de productivité basés sur la diminution des temps de préparation et de mise au point. Un exemple récent illustre parfaitement cette démarche : on sait que la PCR (amplification d'ADN in vitro) est devenue depuis quelques années l'une des opérations les plus quotidiennes de la biologie moléculaire. Cependant, malgré sa simplicité conceptuelle, l'efficacité de l'amplification dépend d'un certain nombre de paramètres qui ne sont pas toujours bien maîtrisés, et il est souvent nécessaire d'optimiser expérimentalement les conditions de l'amplification. La firme américaine Stratagene a repéré ce besoin de mise au point, et propose désormais une trousse d'optimisation de la PCR. Cette trousse offre une combinaison prête à l'emploi de différents tampons et additifs qui permet de tester rapidement une dizaine de conditions différentes d'amplification.

\section{L'automatisation. L'automatisation} est une voie radicalement différente de celle des trousses manuelles. On conçoit facilement qu'elle soit la voie royale pour augmenter la cadence d'opérations répétitives. Il ne s'agit pas alors de faciliter le travail de l'expérimentateur, mais plutôt de "remplacer" celui-ci pour des opérations standardisées. L'opérateur est alors libéré de ce travail répétitif, et peut se consacrer à d'autres travaux à plus forte valeur ajoutée.

Dans certains cas, cette augmentation des cadences a des conséquences économiques importantes, qui apportent à leur tour des potentialités nouvelles à la recherche en biologie. C'est ainsi que la synthèse d'ADN a connu une véritable explosion lorsque les premiers synthétiseurs automatiques sont arrivés sur le marché. En effet, la synthèse manuelle d'ADN est une opération délicate (il s'agit d'une chimie anhydre), longue et fastidieuse. Elle requiert

une main-d'œuvre importante et qualifiée, ce qui entraîne un coût très élevé de l'ADN de synthèse. L'apparition de machines automatiques a rendu économiquement accessible l'utilisation d'ADN de synthèse dans les expériences. Celui-ci est désormais un outil quotidien de la biologie moléculaire, fourni très souvent par des sociétés de service.

Mais l'automatisation n'a pas seulement un impact sur la cadence des opérations répétitives. Elle est aussi efficace pour la diminution des phases de préparation et de mise au point, par la suppression du "coup de main" de l'expérimentateur, et l'offre d'une trousse de réactifs allant de pair avec l'instrument automatique. Il est ainsi plus facile de reproduire des résultats obtenus dans un autre laboratoire quand on achète un appareil automatique que lorsqu'on y implante une technique manuelle. Cependant, l'investissement nécessaire dans l'acquisition d'un tel appareil ne se justifie en général que lorsqu'il y a une certaine cadence à soutenir.

\section{L'automatisation des opérations de routine}

Les opérations de biologie moléculaire, qui ont été automatisées ces dernières années, ou qui sont en cours d'automatisation, sont les différents types de préparation d'acides nucléiques (ADN génomique à partir du sang, de cellules en culture, ou de tissus, $\mathrm{ADN}$ plasmidique ou phagique, $\mathrm{ADN}$ et $\mathrm{ARN}$ de synthèse), l'amplification de l'ADN in vitro par PCR, les techniques d'analyse sur membrane (Southern, Northern, Western et dot blots), le séquençage de l'ADN et certaines analyses d'images. En ce qui concerne l'analyse d'image, la banalisation de l'acquisition d'images vidéo a permis de développer des logiciels d'analyse performants et - en un certain sens - universels. La numérisation de l'image, et les données quantitatives ou qualitatives que l'on peut en extraire sont devenues bien plus accessibles et fiables que celles obtenues avec les méthodes densitométriques. De plus, cela permet de séparer presque complètement l'acquisition de l'image de 


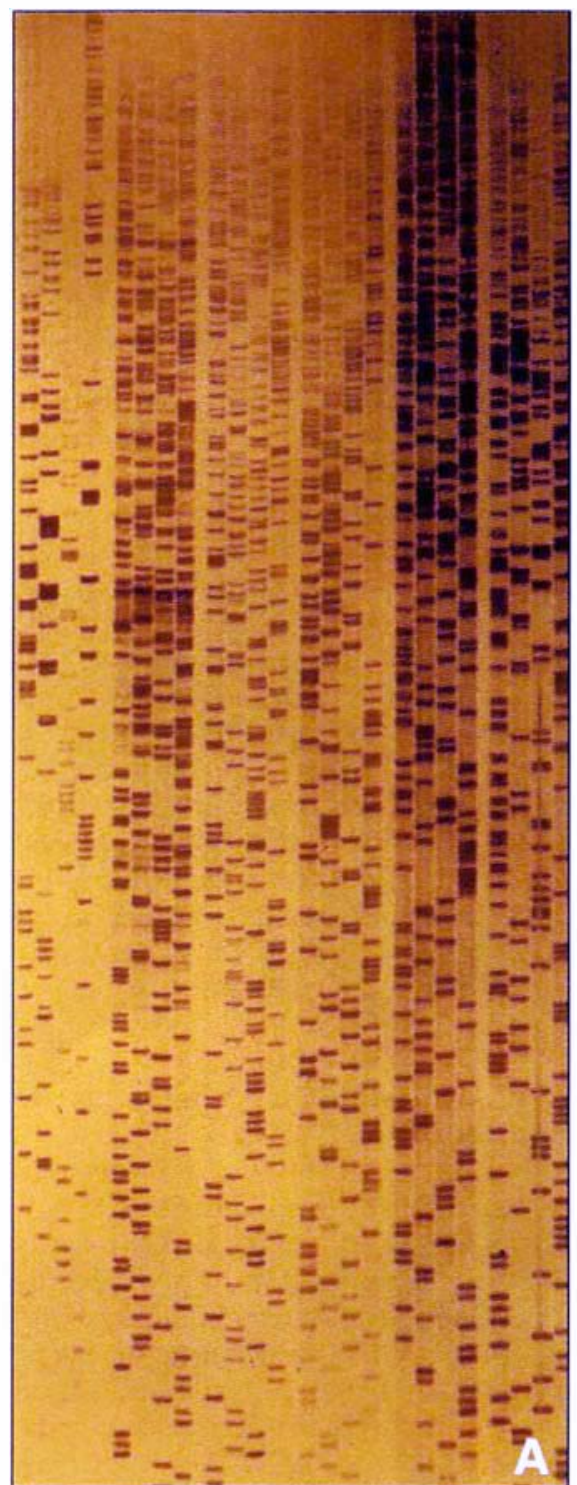

Figure 1. Automatisation du séquençage de I'ADN. A. Autoradiogramme $d^{\prime} u n$ gel de séquence $d^{\prime} A D N$ obtenu par une méthode manuelle. Un tel résultat se lit par groupe de quatre couloirs. La disposition relative de ces bandes les unes par rapport aux autres est traduite en séquence $d^{\prime} A D N$. Il existe maintenant des lecteurs automatiques pour ces autoradiogrammes. B. Séquenceur automatique de la société Applied Biosystems (photo AFM). Les séquenceurs automatiques détectent les fragments d'ADN directement au cours de leur séparation par électrophorèse, sans avoir à passer par un

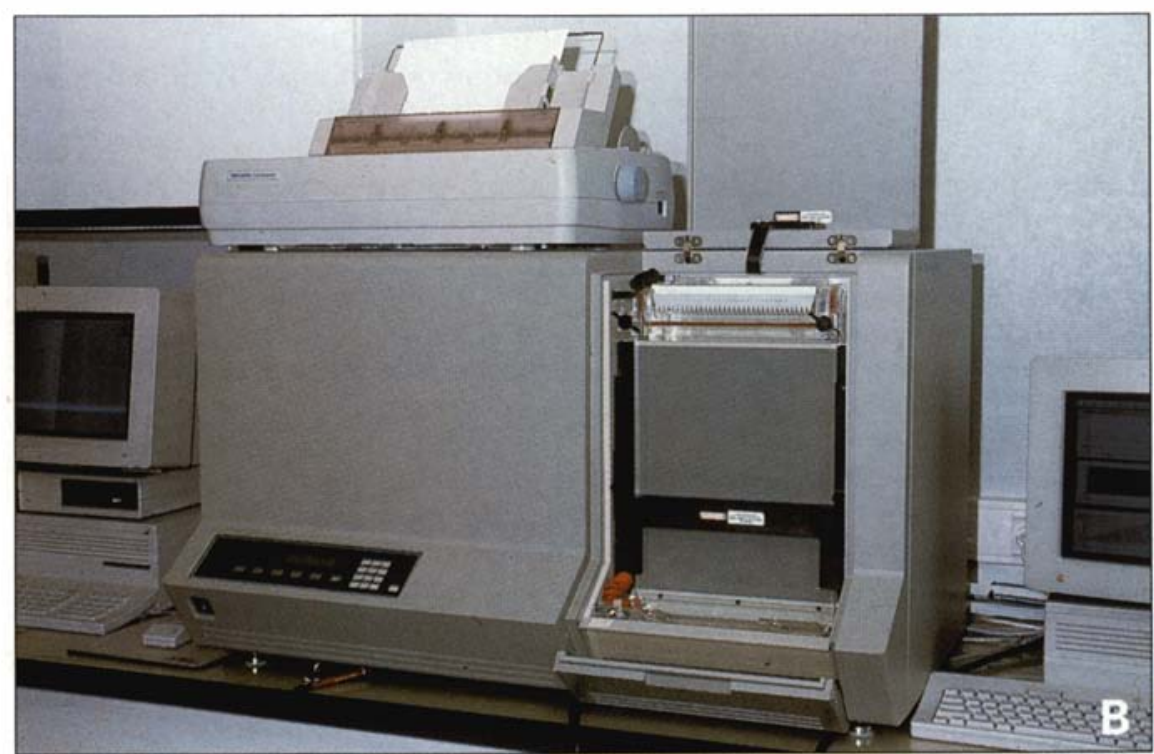

son analyse et interprétation, ce qui n'était pas possible auparavant. Il suffit de changer l'objectif placé devant la caméra pour s'intéresser à des images de l'ordre du micron (cas des hybridations in situ sur chromosome par exemple) ou de plusieurs dizaines de centimètres (cas des techniques d'analyse sur membrane). Les algorithmes de base de reconnaissance de formes et de quantification des signaux sont alors très souvent similaires (reconnaissance de bandes et de points). Il existe ainsi des progiciels d'analyse d'image, intégrant un grand nombre de fonctions, et à partir desquels on pourra programmer de façon relativement simple des algorithmes spécifiques d'une application donnée. Pour donner un exemple, citons le logiciel Optimas de BioScan, devenu ainsi un des progiciels leader dans le monde de la biologie.

L'analyse automatique d'image a donc connu récemment une grande extension en biologie moléculaire. Un exemple assez spectaculaire des progrès accomplis est la lecture automatique des gels de séquence d'ADN; il s'agit de traduire en séquences d'ADN le profil d'électrophorèse, souvent un peu courbé, de milliers de fragments d'ADN s'étalant sur une surface de l'ordre de 30 $\times 40 \mathrm{~cm}$ (figure 1A). Cette traduction prend des heures quand elle est faite manuellement, et nécessite une double lecture. Quand elle est faite à l'aide d'un lecteur automatique, quelques dizaines de minutes suffisent, sans compter que les séquences lues sont fournies directement dans des fichiers informatiques*.

Les systèmes d'analyse d'image sont également très utiles dans le domaine médical. Ainsi, le comptage de particules dans le champ d'un microscope est une technique existant depuis environ dix ans. Elle est appliquée en routine à l'analyse des urines infectées. Aussi, la reconnaissance automatique de formes plus complexes semble désormais possible. La Société Biocom annonce qu'elle pourra très prochainement trier et apparier automatiquement les chromosomes d'un caryotype. Le système distinguera alors entre les échantillons normaux et les échantillons anormaux. L'anatomopatholo-

* Voir par exemple le Basescanner d'Amersham.
Millipore et Biorad proposent des produits équiva-
lents. $\mathrm{m} / \mathrm{s} n^{\circ} 3$, vol. 11, mars 95 


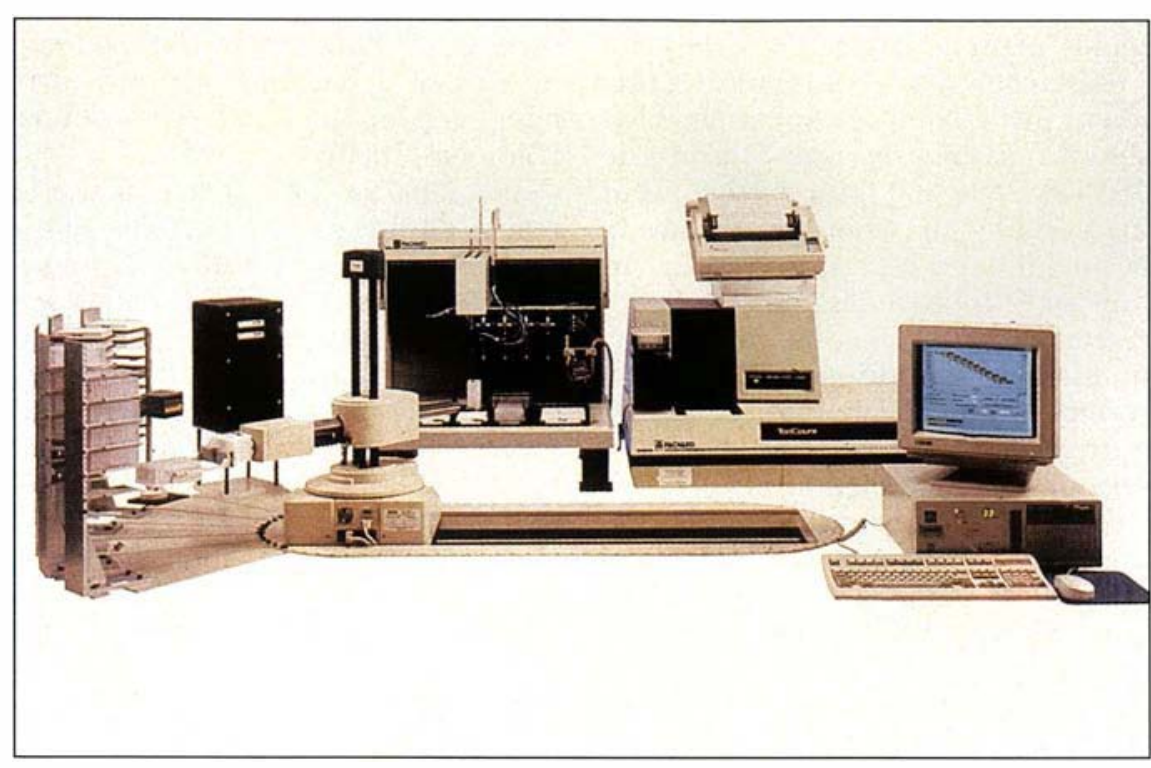

Figure 2. Exemple d'une installation automatique de Zymark. Un bras robot relie une aire de stockage, une station de dilution, et un lecteur de plaque. L'installation est pilotée par un micro-ordinateur (photo Zymark). Voir la plaquette de Zymark "Automatisation du laboratoire", 1994. Zymark, SA, BP 40016, 95911 Roissy Cedex. giste se concentrera sur ces derniers. Son travail en sera grandement facilité (il aura cinq à six fois moins d'échantillons à observer), et son diagnostic en sera également fiabilisé (moins de fatigue; J.L. Weber, communication personnelle). Il faut cependant reconnaître que ce type d'analyse reste difficile, et que les algorithmes utilisés sont encore très loin des performances de l'œil humain.

Mais les difficultés techniques d'automatisation ne sont pas réservées à l'analyse d'image. L'automatisation des méthodes manuelles est rarement une chose aisée..., et demande donc des investissements lourds. On peut distinguer formellement deux voies pour le développement d'un instrument automatique.

1. Automatisation du procédé manuel. La première voie cherche à automatiser le procédé manuel, sans le changer fondamentalement. Par définition, un procédé manuel est toujours composé d'une suite d'étapes, chaque étape utilisant un appareil de laboratoire qui peut être très simple (une pipette, un bain-marie...) ou plus sophistiqué (un diluteur automatique, un lecteur de microplaques...). La démarche de certains industriels, comme Zymark par exemple, consiste tout simplement à robotiser les différentes étapes du protocole à l'aide d'un bras robot. Celui-ci prend les échantillons, les pèse, les emmène à une station de dilution, etc. (figure 2). Il ne s'agit pas vraiment d'un appareil automatique, puisqu'il n'y a pas d'intégration des différentes fonctions dans un même appareil. Il s'agit plutôt d'une installation, dont l'étendue et le coût dépendront du nombre et de la nature des postes desservis par le robot. De fait, on trouve très peu de ces installations dans les laboratoires de recherche. On les trouve plutôt dans les laboratoires de contrôle qualité des industries pharmaceutiques; l'installation est alors conçue au cas par cas, selon les besoins du laboratoire concerné. Quand les étapes successives d'un procédé manuel ne sont pas trop nombreuses ou utilisent toujours les mêmes outils (par exemple des pipettes et une centrifugeuse), leur intégration dans un même appareil est toujours possible. Elle n'est cependant pas toujours heureuse car elle entraîne de nombreux mouvements mécaniques et de fluides qui peuvent être la source de pannes ou de conta-

minations croisées entre échantillons. De plus, cela produit en général des appareils encombrants. Cette démarche a cependant un intérêt certain. Comme le procédé de base est un procédé éprouvé par des années d'expériences dans un grand nombre de laboratoires du monde entier, ses performances et sa fiabilité sont certaines. Le développement de l'appareil pourra donc être relativement rapide, car la partie biochimique du développement sera très courte. Cette voie d'automatisation est en général choisie par les industriels japonais. En témoignent par exemple les appareils impressionnants qu'a développés Seiko pour l'automatisation des réactions de séquence il y a quelques années... appareils peu attrayants qui n'ont pas été commercialisés hors du Japon*. Un autre exemple de cette démarche d'automatisation, qui semble plus réussi, est le préparateur d'ADN $A u$ togen. Distributions de réactifs, pipettages et centrifugations sont combi-

\footnotetext{
* L'usine à séquencer japonaise Huga I suit égale ment cette approche [9]. Les appareils Seiko cités on d'ailleurs été développés en vue de cette installation. L'efficacité de cette installation reste encore à prouver... [10].
} 
nées dans un même appareil qui permet presque tout type de mini-préparation d'ADN. Le proche avenir nous fixera sur le succès commercial de cet appareil, apparu sur le marché il y a environ trois ans, et qui commence à se répandre.

2. Modification des procédés à des fins d'automatisation. La deuxième voie que peut choisir celui qui cherche à développer un appareil automatique consiste à imaginer un procédé nouveau, plus propre à l'automatisation que le protocole manuel. L'intérêt majeur du développement d'un procédé nouveau est d'aboutir à un appareil plus simple, et donc $a$ priori plus fiable. Mais cela engendre la nécessité d'un développement "procédé" qui est en général long, car ses performances doivent au moins égaler celles du procédé manuel, optimisé par des années d'utilisation. C'est la voie qui a été suivie au milieu des années 1980 par les pionniers des séquenceurs automatiques, qui ont imaginé une lecture en ligne des gels de séquence [1113]. Cette lecture en ligne permet d'obtenir les séquences directement dans des fichiers informatiques, sans passer par des manipulations successives du gel qui sont nécessaires au procédé manuel: démoulage du gel, fixation, séchage, autoradiographie, et enfin lecture. Les premiers appareils commerciaux ont été proposés par Applied Biosystems (ABI) et Du Pont à la fin des années 1980 (figure 1B). La société Labimap a également choisi cette démarche d'automatisation pour son extracteur d'ADN génomique [14]. Ici, le procédé de purification de l'ADN génomique a été radicalement modifié par rapport à la méthode traditionnelle afin d'éliminer les étapes de centrifugation et de pipettage. Des étapes de filtration et de dialyse les ont remplacées, qui ne nécessitent pas de mouvements mécaniques. Là encore, le succès commercial de l'appareil dans les années à venir nous renseignera sur le bien-fondé de cette démarche.

Bien que ces appareils automatiques soient attendus par beaucoup, la pé- boratoires est lente*. Trois raisons au moins peuvent être avancées à cette «résistance» des laboratoires. La première est le coût des appareils, bien sûr. Le niveau de prix (disons de 300000 à 800000 francs) est souvent inaccessible au laboratoire conventionnel muni de ses seuls crédits, surtout en Europe où les coûts de maind'œuvre et les coûts de fonctionnement sont disjoints. Pour le directeur d'un tel laboratoire, l'achat d'un appareil de ce type est alors souvent un véritable exploit [15]. La seconde raison, indissociable de la première, est la concurrence des trousses manuelles. Même si celles-ci sont en général chères d'utilisation, aucun investissement n'est nécessaire. Et bien que les trousses manuelles ne libèrent pas le chercheur de ses opérations de routine ni ne lui apportent la reproductibilité de l'automatisation, elles lui permettent d'accélérer sa cadence de façon appréciable. La troisième raison enfin est un relatif échec technique de certains appareils qui ne répondaient pas vraiment à l'attente des utilisateurs. L'extracteur d'ADN d'ABI semble se ranger dans cette catégorie, puisque bon nombre de laboratoires, l'ayant acheté, ne l'utilisent plus. Il ne ferait en fait guère gagner de temps à l'opérateur par rapport aux méthodes manuelles.

Il est, cependant, certain que, peu à peu, les appareils de ce type vont percer dans les laboratoires. Car l'automatisation apporte cadence, fiabilité et reproductibilité, trois éléments essentiels de la productivité.

\section{La place spéciale du liquid handling}

Parmi les opérations de routine utilisées dans les laboratoires, les plus courantes sont certainement les pipettages et les incubations dans des bains-marie ou des étuves. Il est donc tentant de concevoir un appareil qui automatiserait ces fonctions élémentaires ; c'est le concept de «station de

* Il est ici fait exception de la diffusion large et rapide des thermocycleurs, appareils automatisant les cycles d'incubation à différentes températures qui sont utilisés dans la technique de PCR. Ce cas ex ceptionnel, dû à la simplicité et à la puissance de la $P C R$, n'est pas représentatif du mouvement d'ensemble de l'automatisation en biologie moléculaire. travail ", qui serait un appareil à tout faire pour l'opérateur. En biologie moléculaire, Beckman a été le premier à proposer une telle station (Biomeck 1000).

Cependant, ce concept se heurte à la réalité du travail de recherche ; il est vrai qu'il y a des opérations de routine à automatiser, mais les pipettages et incubations sont en général le cour des expériences du chercheur. Or celui-ci fait rarement la même expérience un grand nombre de fois. Il lui faudra donc reprogrammer sa station de travail à chaque expérience. Une telle programmation nécessite en général une mise au point assez longue. La station de travail ne fera par conséquent pas gagner de temps à ce chercheur.

Ce concept de station de travail conduit donc à des gains de productivité uniquement par l'accélération de la cadence d'opérations répétitives, et non par la diminution des phases de préparation et de mise au point des expériences. C'est pourquoi il n'est intéressant que pour les opérations très répétitives, comme le séquençage de l'ADN à grande échelle ou des tests de criblage standardisés. Ces stations de travail se retrouvent donc dans des laboratoires dont l'activité est focalisée sur un très petit nombre de techniques, comme certains laboratoires d'industries pharmaceutiques ou agro-alimentaires, ou les gros centres de recherche sur le génome humain. Mais elles sont extrêmement rares dans les laboratoires de recherche conventionnels, où leur utilité est limitée.

Il faut cependant noter que cette situation évolue. Les logiciels d'interface s'améliorent, et la programmation devient de plus en plus facile pour l'utilisateur**. Le jour où cette programmation sera vraiment facile, ne nécessitant pas plusieurs expériences "à blanc" pour tester le programme fait, il n'est pas impossible que ce concept d'appareil "à tout faire"se développe. Mais il ne remplacera

** A l'heure actuelle, la Société Beckman lance une nouvelle version de sa station de travail, le Biomek 2000. La facilité et la convivialité du logiciel de programmation sont les arguments majeurs développés par la force de vente. 
probablement jamais l'opérateur humain pour certains travaux d'investigation qui nécessitent de modifier l'enchaînement des étapes initialement prévues en fonction de difficultés observées avec son échantillon (besoin d'une purification supplémentaire de l'ADN, pipettage plus lent à cause d'une forte viscosité inattendue, etc.)

Les possibilités nouvelles apportées par l'instrumentation automatique

Nous avons déjà montré ci-dessus à propos de la synthèse d'ADN comment l'automatisation d'un procédé pouvait apporter des possibilités nouvelles aux chercheurs, et leur permettre des stratégies d'investigation plus puissantes. Dans les lignes qui suivent, nous nous efforcerons de discerner les nouvelles possibilités qui émergent aujourd'hui (ou émergeront bientôt) grâce à l'instrumentation, et quelques orientations de développements futurs.

L'augmentation de la cadence suffit parfois à elle seule à rendre accessibles certaines stratégies jusqu'ici impossibles. Le domaine de la biologie où cela est le plus visible aujourd'hui est sans doute celui de la génétique, qu'elle soit humaine, animale ou végétale. Les gros centres du génome utilisent les même techniques que les laboratoires traditionnels de génétique, mais sur une échelle bien plus grande grâce à une organisation du travail de production (et non de recherche) et à l'appel massif à l'instrumentation automatique. Nous avons cité l'exemple des cartes génétiques et physiques réalisées au Généthon. Nous pouvons illustrer cela également en évoquant la mise au point du séquençage à grande échelle. La recherche de gènes dans une région d'ADN physiquement déterminée est désormais envisagée par séquençage systématique de la région considérée, et analyse de la séquence obtenue. Le projet européen de séquençage du génome de la levure a déjà montré les fruits d'une telle approche $[16,17]$. Celle-ci devient également possible chez les mammifères $[18,19]$. Cependant, cette approche n'est pas accessible pour le moment à un laboratoire conventionnel isolé.
L'accès à l'information et son traitement

La place de l'informatique est grandissante en biologie, et cette évolution n'est pas terminée. Toute personne effectuant un travail de recherche doit désormais utiliser des bases de données, qu'elles soient bibliographiques, de séquences d'ADN ou de protéine, ou d'autres types d'informations. Le nombre et la taille de ces bases de données grandissent sans cesse, chaque centre branchant son serveur sur le réseau scientifique international Internet [20]. L'utilisation de ces bases de données apporte une quantité de connaissances inaccessibles autrement. Elle est devenue indispensable à tout travail de recherche, sous peine de perdre du temps ou de passer à côté de découvertes significatives. Inutile de dire que la constitution de ces bases de données et leur consultation nécessitent des moyens informatiques de plus en plus performants. Mais il devient essentiel de coordonner ces différentes bases de données pour une meilleure efficacité des investigations, et beaucoup de progrès sont encore à faire en ce domaine pour rendre les recherches dans les différentes bases de données faciles et rapides pour le chercheur non spécialiste.

Mais il s'agit là d'un travail qui ne rentre pas dans le cadre du développement d'appareils de laboratoire. Il s'agit plutôt de la mise en place de réseaux, nécessitant des investissements lourds en hardware et en software. Cela devrait relever plus des institutions publiques que des industriels, même si la création ou la gestion de ces réseaux sont confiées à des entreprises privées.

\section{Le séquençage de l'ADN}

Cela est et restera l'un des enjeux majeurs pour les industriels s'intéressant à l'instrumentation automatique pour la biologie moléculaire. En effet, le séquençage est l'analyse la plus fine que l'on puisse faire de l'ADN, même si l'on ne sait pas encore toujours en retirer l'information souhaitée. Il est utile, non seulement pour les gros projets de séquençage abondamment annoncés dans les médias, mais aussi comme outil quotidien de contrôle dans tous les laboratoires. Si cela devenait économiquement accessible, il pourrait être aussi utilisé à des fins diagnostiques.

Il y a quelques années, les méthodes qui allaient révolutionner la façon de séquencer l'ADN foisonnaient. On parlait d'une lecture directe des bases de l'ADN par microscopie à champ proche $[21,22]$. Le DOE américain travaillait avec acharnement sur une méthode ambitieuse, fondée sur une digestion exonucléolytique d'une molécule unique d'ADN dont chaque base serait fluorescente, et dont la détection serait réalisée au fur et à mesure de la digestion [23, 24]. On évoquait encore la possibilité de séparer les produits des réactions de séquence par de nouvelles techniques de spectrométrie de masse, 10000 ou 100000 fois plus rapides que l'électrophorèse en gel d'acrylamide [25]. D'autres techniques étaient également envisagées. Il est à noter que tous ces projets étaient dirigés vers le séquençage à grande échelle, nécessaire aux projets Génome, et non vers l'amélioration de l'outil quotidien d'un laboratoire de recherche conventionnel.

Aujourd'hui, après deux à quatre ans de travail, les espoirs mis dans ces méthodes sont beaucoup plus mesurés, ou ont même disparu. Des difficultés techniques très importantes ont été rencontrées lors des travaux, ainsi que parfois des limitations physiques (des phénomènes quantiques commencent à prédominer quand on s'intéresse à la détection fluorescente d'une molécule unique, par exemple). On parle désormais plus volontiers d'évolution des stratégies de séquençage et des séquenceurs actuels dans les quatre années à venir que d'un bouleversement véritable de ces méthodes. L'évolution consiste en une multiplicité d'améliorations portant sur la synthèse des amorces pour les réactions de séquence, sur les gels d'électrophorèse sur la vitesse et la sensibilité des détecteurs utilisés dans les séquenceurs, et sur les logiciels de lecture en ligne des réactions de séquence.

Il existe, cependant, une méthode nouvelle de séquençage en cours de 
développement, appelée le séquençage par hybridation, et qui consiste à hybrider le fragment d'ADN à analyser avec un très grand nombre d'oligonucléotides de synthèse. L'analyse des hybridations permet de remonter à la séquence initiale [26, 27]. Plusieurs difficultés techniques restent à résoudre pour que cette méthode soit vraiment opérationnelle, mais elles semblent jusqu'à présent surmontables. Il est cependant probable que la méthode sera d'abord utilisée pour l'analyse de gènes connus (diagnostic génétique par exemple), car cette application demande un nombre nettement plus restreint d'oligonucléotides que le séquençage direct d'ADN inconnu.

\section{Autres domaines}

On se rappelle la révolution amenée par la PCR pendant les années 1980. Ce procédé d'amplification de l'ADN in vitro, à la fois simple, puissant et souple, a changé profondément la façon de pratiquer la biologie moléculaire. Une telle révolution est évidemment difficile à prédire, mais il ne semble pas qu'il y ait quelque chose de cette ampleur en cours à l'heure actuelle. On peut, cependant, noter que, dans le domaine de la biochimie et de l'immunochimie, les biocapteurs capables de suivre des interactions moléculaires sans marquage des molécules sont peut-être en train d'apporter une révolution de ce type. Il s'agit des appareils BIAcore de Pharmacia, et BIOS-1 de Artificial Sensing Instrument (figure 3). Une cartographie des épitopes reconnus par une série d'anticorps dirigés contre un antigène donné peut être réalisée en deux semaines avec ces appareils, avec acquisition des constantes cinétiques des liaisons et sans crainte de déformations des anticorps ou des épitopes dues au marquage des molécules $[28,29]$. Ce travail nécessite des mois par les méthodes traditionnelles, qui ne donnent que rarement accès aux constantes cinétiques. La première génération de ces appareils n'est pas bien adaptée aux besoins de la biologie moléculaire, car la sensibilité en est un peu faible. Mais la seconde gé-

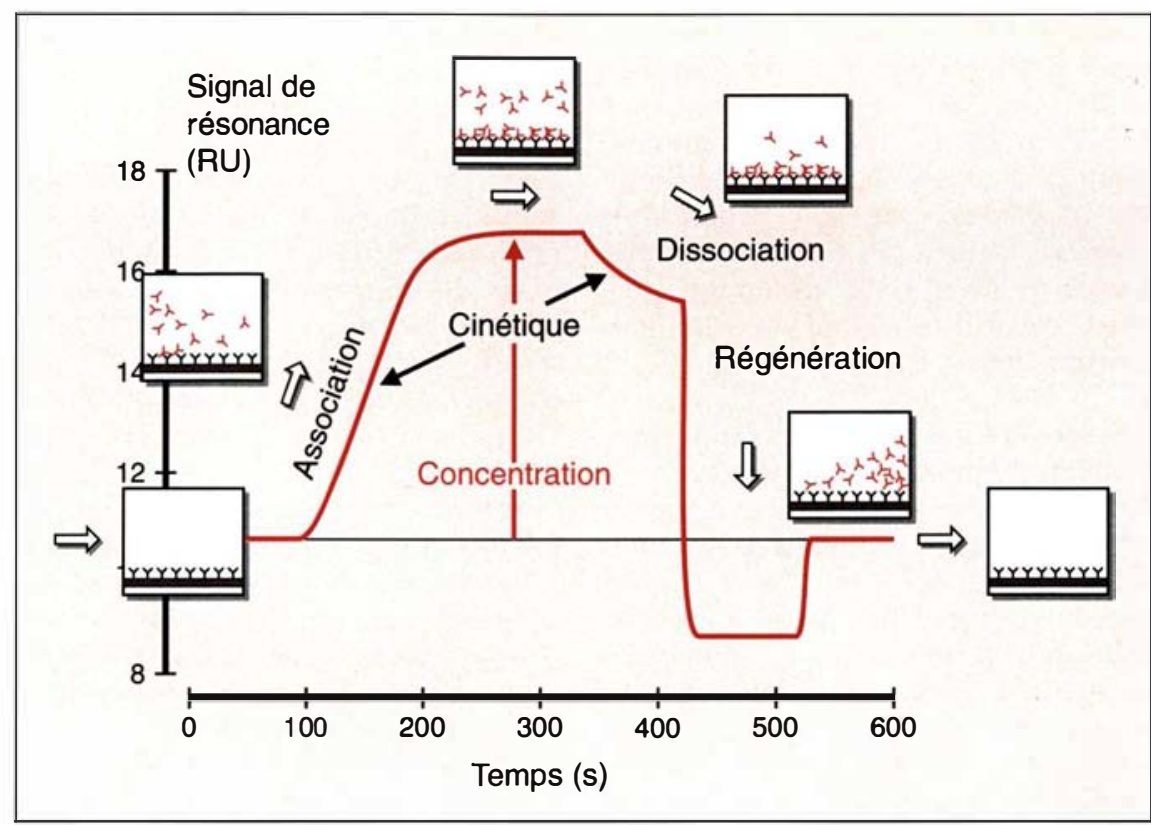

Figure 3. Résultats obtenus par un biocapteur. Au temps zéro de l'expérience, une molécule d'intérêt (appelée ici récepteur) se trouve fixée sur la surface du biocapteur. Cette surface est continuellement baignée par un flux de tampon. Le biocapteur mesure un signal optique qui est proportionnel à la masse présente sur la surface. Le signal optique engendré par la présence du récepteur forme alors la ligne de base de la courbe de résultat (marquée sur la figure par une flèche horizontale). Au temps 100 s est injecté sur la surface un flux de tampon contenant une molécule (appelée ici ligand) dont on veut étudier la liaison avec le récepteur. Au fur et à mesure que le ligand s'accroche au récepteur, le signal optique augmente, jusqu'à atteindre un plateau correspondant à l'équilibre thermodynamique ligand-récepteur. Une fois ce plateau atteint, on fait à nouveau passer sur la surface du tampon sans ligand, le ligand se dissocie alors du récepteur, et le signal optique suit la décroissance exponentielle de cette dissociation. Les trois phases de cette courbe (association, dissociation, et plateau) permettent de déterminer les constantes cinétiques d'association et de dissociation, la constante d'équilibre, et la concentration du ligand. La surface du biocapteur est ensuite régénérée, et un nouveau ligand peut être étudié. Un cycle complet prend en général de l'ordre de 10 minutes. Des interactions moléculaires multiples peuvent être étudiées de la même façon.

marché, nettement plus sensible*. Nul doute alors que les études d'interaction $\mathrm{ADN}$-protéines comme celle de Fischer et al. [30], ou d'interaction protéine-protéine vont se multiplier.

\section{Conclusion}

L'évolution de la biologie vers "plus d'instruments" que nous avons décrite au début de cet article se poursuivra sans doute dans les prochaines

* Il s'agit par exemple du BIAcore 2000 de la Société Pharmacia. années. «Produire plus» reste une nécessité vitale pour les laboratoires, et les instruments disponibles sur le marché sont encore loin de remplir toutes les possibilités d'automatisation qui existent. Cependant, il me semble que la place du laboratoire traditionnel n'est pas menacée par cette évolution et par l'apparition des gros centres dédiés aux projets Génome. Certains se plaisent à dire que la biologie entre dans l'ère de la big science, au même titre que la physique des particules par exemple. Je ne pense pas que l'analogie soit vrai- 
ment pertinente. Non seulement parce que les sommes mises en jeu ne sont pas encore du même ordre, mais aussi et surtout parce que les gros centres et les gros projets tendent à l'acquisition rapide de données de base, devant servir d'outil élémentaire à la recherche future. Il ne s'agit donc pas de monter des bancs d'expériences coûteux répondant à une question scientifique particulière, comme cela est le cas de la recherche sur la masse des neutrinos, ou sur la fission nucléaire contrôlée. Les expériences cherchant à décortiquer les mécanismes du vivant resteront encore de très nombreuses années à l'échelle du laboratoire traditionnel, sachant aller chercher les techniques et les instruments nécessaires là où ils se trouvent. L'instrumentation permettra "seulement" de mettre en place ces expériences beaucoup plus rapidement. Il faut, cependant, veiller à ce que les données engendrées par les gros centres de type industriel - dont certains sont de statut privé, et la plupart aux États-Unis - restent raisonnablement accessibles aux laboratoires de recherche, et que ceux-ci puissent s'équiper convenablement en équipements mi-lourds. Autrement, la recherche française perdra sa compétitivité face à ses concurrentes, et d'abord, bien sûr, face aux États-Unis

\section{TIRÉS À PART}

F. Ginot.

$m / s n^{\circ} 3$, vol. 11, mars 95

\section{Summary}

Automatic equipment in molecular biology

The techniques and equipment used in molecular biology are evolving rapidly. This article tries to draw some features of this evolution through the concept of productivity improvement. Two areas are distinguished where laboratories tend to increase their productivity: the rate of repetitive operations and the time spent in setting up new techniques. The role and trends of automatic equipment in these areas are discussed. Also, it is shown that, when automation makes things really easier and cheaper, the way of doing research is changed.

\section{Remerciements}

Je remercie vivement Karin Costes et Laurence Ginot pour leur lecture attentive de ce manuscrit et leurs commentaires, le Dr Weber pour ses informations sur l'analyse d'image en clinique, et Christine Rémy pour la mise en forme du manuscrit. Les sociétés Zymark et Pharmacia ont fourni des illustrations.

\section{F. Ginot}

Responsable recherche et développement. Labimap SA. 59, rue Pierre-Curie, ZI des Gâtines, BP 3, 78373 Plaisir Cedex France. Adresse actuelle : Généthon, 1, rue de l'Internationale, BP 60, 91002 Évry Cedex, France.

\section{RÉFÉRENCES}

1. Rocher YA. La recherche française estelle bien équipée ? La Recherche $1989 ; 216$ : 1539-42.

2. Callou M, Leydesdorff L. La recherche française est-elle en bonne santé ? $L a R e-$ cherche 1987 ; 18 : 412-3.
3. Gorin MB, Yancey SB, Cline J, Revel JP, Horwithz I. The major intrinsic protein (MIP) of the bovine lens fiber membrane: characterization and structure based on cDNA cloning. Cell $1984 ; 39: 49-59$.

4. Lynch DC, Zimmerman TS, Collins CJ, Brown M, Morin MJLEH, Livingston DM. Molecular cloning of cDNA for human Willebrand factor: authentication by a new method. Cell 1985 ; 41 : 49-56.

5. Hake LE, Richter JD. CPEB is a specificity factor that mediates cytoplasmic polyadenylation during xenopus oocyte maturation Cell $1994 ; 79: 617-27$

6. Schaffer JE, Lodish HF. Expression cloning and characterization of a novel adipocyte long chain fatty acid transport protein. Cell $1994 ; 79: 427-36$

7. Jordan B. Généthon : la réussite d'un pari. médecine/sciences $1992 ; 8: 1102-5$.

8. Weissenbach J, Gyapay G, Vignal A, Dib C, Benigni X, Vaysseix G, Morissette J. Cartographie génétique à l'échelle industrielle. $R B M 1992 ; 14$ : 146-50.

9. Endo I. A human genome analysis system (HUGA-I) developed in Japan. Adv Biochem Eng Biotech 1992 ; 46 : 103-10.

10. Jordan B. Les HGM se suivent... et ne se ressemblent pas. médecine/sciences $1994 ; 10$ 92-5.

11. Smith LM, Sanders JZ, Kaiser RJ, Hu ghes $\mathrm{P}$, Dodd $\mathrm{C}$ Connell $\mathrm{CR}$, Heiner $\mathrm{C}$ Kent SBH, Hood LE. Fluorescence detection in automated DNA sequence analysis. Nature $1986 ; 321$ : 674-8.

12. Prober JM, Trainor GL, Dam RJ, Hobbs FW, Robertson CW, Zagursky RJ, Cocuzza AJ, Jensen MA, Baumeister $K$ A system for rapid DNA sequencing with fluorescent chain-terminating dideoxynucleotides. Science 1987 ; 238 : $336-41$.

13. Ansorge W, Sproat BS, Stegemann J Schwager C. A non-radioactive automated method for DNA sequence determination. $J$ Biochem Biophys Methods 1986 ; 13 : 315-23.

14. Hache J. Un robot pour Southern. Bio futur 1992 ; 14-6.

15. Coutures JP. La course d'obstacles. Lo Recherche 1990 ; 225 : 1160-1.

16. Oliver SG, Vanderaart QJM, AgostoniCarbone ML, Aigle M, Alberghina L, Alexandraki D, Antoine G, Anwar R, Ballesta JPG, Benit P, et al. The complete DNA sequence of yeast chromosome-III. Nature quence of yeast
$1992 ; 357: 38-46$.

17. Dujon B, Alexandraki D, Andre B, Ansorge W, Baladron V, Ballesta JPG, Banrevi $A$, Bolle PA, Bolotinfukuhara $M$, Bossier $P$, et al. Complete DNA sequence of yeast chromosome XI. Nature 1994 ; 369 : 371-8.

18. Buck D, Bailey J, Baron L, Bates G, Baxendale S, Berks M, Conroy D, Dods- 
worth S, Fowler J, Gardner A, et al. Sequencing and analysis of a 2 megabase human cosmid contig containing the Huntington's disease gene. Genome sequencing and analysis conference VI. Mary Ann Liebert, Inc. 1994 ; 46.

19. Muzny DM, Lu F, Timms K, Andersson B, Shen Y, Gibbs RA. Shotgun sequencing of Xq28 from FraxA to IDS. Genome sequencing and analysis conference VI. Mary Ann Liebert Inc. $1994 ; 48$.

20. Aldhous P. Managing the genome data deluge. Science 1993 ; 262 : 502-3.

21. Jordan B. Le tunnel séquencera-t-il le génome? médecine/sciences $1990 ; 6$ : 1007-9.

22. Hansma HG, Weisenhorn AL, Gould SAC, Sinsheimer RL, Gaub HE, Stucky GD, Zaremba CM, Hansma PK. Progress in sequencing deoxyribonucleic acid with an atomic force microscope. I Vac Sci Technol 1991 ; $\mathrm{B9}$ : 1282-4.

23. Jett J, Keller R, Martin J, Moysis RK, Shera EB. Method for rapid base sequencing in DNA and RNA. Brevet International 1989 ; WO $89 / 03432$.

24. Lloyd D, Fairfield FR, Harger CA, Jett JH, Keller RA, Hahn JH, Krakowski LA Marrone BL, Martin JC, Nutter HL, Ratliff RL, Shera EB, Simpson DJ, Soper SA. Rapid DNA sequencing based upon single molecule detection. GATA 1991; $8: 1-7$.

25. Jacobson KB, Arlinghaus HF, Buchanan MV Chen CH, Glish GL, Hettich RL, McLuckey SA. Applications of mass spectrometry to DNA sequencing. GATA 1991; 8 : 223-9.

26. Khrapko KR, Lysov YP, Khorlyn AA Shick VV, Florentiev VL, Mirzabekov AD. An oligonucleotide hybridization approach to DNA sequencing. FEBS Lett 1989; 256: 118-22.

27. Mirzabekov $\mathrm{AD}$. DNA sequencing by hybridization - a megasequencing method and a diagnostic tool? Trends Biol Tech 1994; 12: $27-32$.

28. Johne B, Gadnell M, Hansen K. Epitope mapping and binding kinetics of monoclonal antibodies studied by real time biospecific interaction analysis using surface plasmon resonance. I Immunol Methods 1993; $160: 191-8$

29. Dubs MC, Altschuh D, Van Regenmortel M. Mapping of viral epitopes with conformationally specific monoclonal antibodies using biosensor technology. I Chromatogr 1992 ; 597 : 391-6.

30. Fisher RI, Fivash M, Casas FI, Erickson JW, Kondoh A, Bladen SV, Fisher C, Watson DK, Papas T. Real-time DNA binding measurements of the ETS1 recombinant oncoproteins reveal significant kinetic differences between the $\mathrm{p} 42$ and $\mathrm{p} 51$ isoforms. Protein Sci 1994 ; 3 : 257-66. 